\title{
Analytische Perspektiven auf Interaktion: Eine Rahmung ${ }^{1}$
}

\section{Einleitung}

Die empirische Untersuchung menschlicher Interaktion hat sich in der Linguistik im deutschsprachigen Raum in Folge der Rezeption der Conversation Analysis (Sacks/Schegloff/Jefferson 1974) seit Ende der 1960er Jahre etabliert und ausdifferenziert. Die inzwischen erreichte Ausdifferenzierung spiegelt sich in den Selbstbezeichnungen der unterschiedlichen Ansätze wider: (ethnomethodologische) Konversationsanalyse, (linguistische) Gesprächsanalyse, (kritische) Diskursanalyse, funktionale Pragmatik, interaktionale Linguistik, interpretative Soziolinguistik, Interaktionsanalyse, multimodale Interaktionsanalyse etc. Um diese Vielfalt begrifflich wieder zu homogenisieren, hat sich die übergeordnete Bezeichnung „Gesprächsforschung“ etabliert. Für alle Ansätze, die in diesem Zusammenhang mit durchaus unterschiedlichen Erkenntnisinteressen arbeiten, ist folgende Gemeinsamkeit konstitutiv: Sie alle haben als empirische Grundlage Dokumente authentischer Interaktion aus sehr unterschiedlichen Situationen. Diese bestehen teils aus Tondokumentationen und erlauben die Analyse der verbalen Interaktionsanteile, teils und in zunehmendem Maße - sind das auch Videoaufzeichnungen, welche die Analyse der faktischen multimodalen Komplexität von Interaktion ermöglichen. Auch die Analysen des hier vorliegenden Bandes sind mit unterschiedlicher Verankerung in diesem ausdifferenzierten empirischen Forschungsfeld verortet. Wie genau diese Verankerung aussieht, wird jeweils zu Beginn der vier Fallanalysen dargestellt (siehe unten).

Es geht jedoch nicht um die Darstellung der den vier analytischen Perspektiven zugrundeliegenden Ansätze. Das Ziel des Bandes ist vielmehr die Präsentation unterschiedlicher analytischer Perspektiven bei der Analyse eines gemeinsamen Interaktionsdokumentes. Ein etwa dreiminütiger Ausschnitt aus einer Unterrichtsstunde wird sowohl aus der Perspektive der „Grammatik der gesprochenen Sprache“, der ,interpretativen Soziolinguistik“, der „ethnografischen Interaktionsanalyse“ sowie aus Sicht der „multimodalen Interaktionsanalyse" untersucht. Es ist dabei methodisch indiziert, dass die jeweiligen Fallanalysen in vollständiger methodischer Autonomie durchgeführt werden und bei ihrer analytischen Rekonstruktionsarbeit keinerlei Bezüge zu den andern Perspektiven herstellen. Jede Perspektive führt vielmehr die für sie konstitutiven Fragestellungen, methodischen Vorgehensweisen und Analyseinstrumente vor. Dies soll die ,methodische Reinheit" der jeweiligen Perspektiven garantieren und ihre spezifischen Erkenntnismöglichkeiten und Erkenntnisgrenzen sichtbar machen.

Die vier Perspektiven auf Interaktion weisen trotz ihrer methodisch motivierten Autonomie eine gemeinsame grundlagentheoretische Basis auf. Sie sind interaktionistisch in ihrer Grundorientierung, operieren auf der Basis einer auf die Konversationsanalyse zurückgehenden konstitutionsanalytischen Methodologie und betrachten Interaktion als gemeinsame Herstellung der Beteiligten (Garfinkel 1967). Diese Gemeinsamkeiten ermöglichen es, nicht nur dasselbe Interaktionsdokument zu analysieren, sondern dabei auch einen

Aufgrund der Umfangsvorgabe des Sonderheftes erfolgt die Darstellung an vielen Stellen in sehr komprimierter Weise. Teilweise konnten dadurch nicht alle relevanten Sachverhalte präsentiert werden. 
gemeinsamen analytischen Fokus zugrunde zu legen. Das übereinstimmende Ziel der vier Perspektiven besteht in der Rekonstruktion von zentralen Aspekten der Interaktionskonstitution aus Sicht des spezifischen Erkenntnisinteresses der jeweiligen Perspektive. Diese Übereinstimmung bezieht sich auch auf die Art und Weise der Gegenstandskonstitution, welche die Rekonstruktionsarbeit der vier Perspektiven ,triggert“. Die Analyseaspekte werden aus der Auseinandersetzung mit den Strukturen des Interaktionsdokumentes entwickelt und nicht subsumtionslogisch durch übergeordnete und den Daten externe theoretische Konzepte.

Die Analyse desselben Interaktionsdokumentes aus vier eigenständigen Perspektiven mit einem gemeinsamen, grundlagentheoretisch motivierten Erkenntnisinteresse ist im deutschsprachigen linguistischen Forschungskontext ein Novum. Der vorliegende Band unterscheidet sich in dieser Hinsicht grundlegend von gängigen Bemühungen um Sichtbarmachung der Eigenständigkeit, Leistungsfähigkeit und der Gemeinsamkeiten unterschiedlicher empirischer Analysemethoden. Dies gilt ganz offensichtlich für klassische Übersichtsdarstellungen, wie etwa den Band „Approaches to discourse“ (Schiffrin 1994). Hier werden sehr unterschiedliche und hinsichtlich ihrer theoretischen Grundlagen und den damit zusammenhängenden Erkenntnisinteressen teils sehr heterogene Ansätze vorgestellt: Sprechakttheorie, Interaktionale Soziolinguistik, die Ethnografie der Kommunikation und Variationsanalyse. Dieser Überblicksdarstellung liegt - bei der beschriebenen Bandbreite der dargestellten Ansätze nicht erstaunlich - kein gemeinsames Interaktionsdokument zugrunde.

Der Band unterscheidet sich jedoch hinsichtlich seiner grundlagentheoretischen Konsistenz und methodologischen Affinität der Perspektiven auch von zunächst etwas näher liegenden Versuchen, wie beispielsweise dem Band „Gesprächsforschung im Vergleich“ (Sucharowski 1985). Hierbei handelt es sich um eine Sammlung von insgesamt sieben Analysen, die als gemeinsame empirische Grundlage ein umfangreiches Transkript und den Videomitschnitt einer „Bonner Runde“ haben. Die Analysen weisen jedoch bewusst keinen gemeinsamen Analysefokus auf. Vielmehr wird die empirische Grundlage genutzt, um sehr unterschiedliche und voneinander gänzlich unabhängige Fragestellungen zu bearbeiten: Das Interaktionsdokument dient beispielsweise dazu, ein ,gesprächsanalytisches Schichtmodell“" (Sager 1985) zu entwickeln, die Bonner Runde als Kommunikationsform zu rekonstruieren (Hoffmann 1985) oder kommunikative Verfahren wie „Einwürfe“ zu analysieren (Fiehler 1985).

Im Unterschied dazu verfolgen wir das Ziel, die spezifischen Leistungen, Erkenntnismöglichkeiten und Grenzen der vier Perspektiven im unmittelbaren Vergleich bei der Analyse desselben - wesentlich kleineren - Ausschnitts aus einem Unterrichtsgeschehen zu verdeutlichen. Wir wollen dabei zeigen, dass die perspektivierte Analyse des Ausschnitts unweigerlich mit einer aspektualisierenden Reduktion der faktischen Komplexität von Interaktion erkauft werden muss. Zweitens - und im gewissen Sinne gegenläufig hierzu - wollen wir jedoch die Frage stellen: Kann die durch die methodische und erkenntnisbezogene Autonomie der vier Perspektiven motivierte Reduktion interaktiver Komplexität im Bezug aufeinander wieder aufgehoben werden oder existiert der Erkenntnisgewinn nur im relativ engen Autonomierahmen des jeweiligen Ansatzes? Drittens interessieren wir uns für die Reflexion der de-facto-methodologischen Bearbeitung allgemeiner, ansatzunabhängiger und forschungslogisch notwendiger Operationen, die sich aus den gemeinsamen grundlagentheoretischen Übereinstimmungen ergeben. 
Wir haben uns dazu entschieden, die Fallanalysen von den beiden letzten Erkenntnisaspekten zu entlasten und die Beschäftigung an einen externen Experten zu übertragen. Es war uns wichtig, dass dieser Experte selbst nicht durch eine der präsentierten analytischen Perspektiven belastet diesen beiden Aspekten nachgehen konnte. Die Reflexion der Erkenntnismöglichkeiten und Grenzen, der Unterschiede und der Gemeinsamkeiten sollte auf der Basis der vorliegenden Fallanalysen erfolgen. Dabei ist uns folgender Fokus wichtig: Das zentrale Erkenntnisinteresse des Unternehmens ,analytische Perspektiven auf Interaktion" richtet sich nicht auf Unterschiede und Gemeinsamkeiten im theoretischen Bereich, sondern fokussiert das analytische Potenzial der Perspektiven bei ihrer konkreten Anwendung. Dazu ist es jedoch notwendig, die Perspektiven bei der Analyse desselben Interaktionsdokumentes beobachten und miteinander vergleichen zu können.

Wir verbinden mit der Auswahl genau der vier analytischen Perspektiven keinerlei wertende Abgrenzung von anderen Ansätzen. Die konkrete Auswahl orientiert sich einerseits an Kriterien eines ,minimalen Kontrastes“, durch den eine gewisse Nähe der Perspektiven gewährleistet wird. Sie ist andererseits Ausdruck der persönlichen wissenschaftlichen Kompetenzen und Vorlieben der Autoren. Im Rahmen der gegebenen Selektionsnotwendigkeit können grundsätzlich nicht alle existierenden Ansätze in ihrer Arbeitsweise präsentiert werden. Die Verortung der Perspektiven im Kontext der inzwischen ausdifferenzierten Forschungslandschaft zur Analyse menschlicher Interaktion erfolgt zu Beginn der Fallanalysen. In dieser Einleitung wollen wir uns nachfolgend auf die Beschreibung und Aufbereitung der gemeinsamen empirischen Grundlagen konzentrieren, die den Fallanalysen zugrunde liegen.

\section{Gemeinsame Grundlagen}

Wir haben einleitend auf die Gemeinsamkeit der empirischen Grundlagen und des zentralen konstitutionsanalytischen Erkenntnisinteresses als Konsistenzkriterien der vier Fallanalysen verwiesen. Wir haben jedoch bewusst darauf verzichtet, den Ausschnitt des Interaktionsdokumentes im Sinne einer verbindlichen Festlegung für die Analyse einzugrenzen. Vielmehr haben wir den analytischen Perspektiven die Möglichkeit eröffnet, im Rahmen der gemeinsamen empirischen Grundlagen einen ihren jeweiligen Erkenntnisinteressen aussagekräftigen oder prototypisch geeigneten Ausschnitt für die Rekonstruktionsarbeit in Auseinandersetzung mit den Daten zu entwickeln. Die Art und Weise, in der sich die vier Fallanalysen bei der Gegenstandskonstitution und der analytischen Rekonstruktionsarbeit auf die gemeinsamen empirischen Grundlagen und auf die intersubjektiv gültige Strukturbeschreibung beziehen, und die Art und Weise, wie sie dort beschriebene Aspekte für ihr Erkenntnisinteresse interpretieren und in ihre eigene Begrifflichkeit überführen, ist bereits wesentlicher Ausdruck ihrer Eigenständigkeit.

Es ist nicht die Aufgabe der Analysen, den oben präsentierten Ausschnitt von Anfang bis Ende sequenzanalytisch zu rekonstruieren. Es geht vielmehr darum, erkenntnisspezifische Untersuchungsschwerpunkte zu setzen. Es soll dadurch deutlich werden, dass die Auswahl der in den Fallanalysen untersuchten Teile des Gesamtausschnittes bereits das Ergebnis der perspektivengebundenen Auseinandersetzung mit den Daten darstellt. Dieselbe Datengrundlage führt also nicht zwangsläufig auch zur Konstitution desselben Untersuchungsgegenstandes. Die Fallanalysen verorten sich in einer knappen Einleitung im oben skizzierten Forschungsfeld und stellen dabei ihre theoretischen und methodischen Grundlagen dar. Sie entwickeln ausgehend von dieser Beschreibung ihren Untersuchungs- 
gegenstand und ihr zentrales datenbezogenes Erkenntnisinteresse. Darüber hinaus sind die Fallanalysen weitgehend frei von literaturbasierten Forschungsüberblicken oder literaturbezogenen Diskussionen. Ihre primäre Aufgabe ist es, die methodische Arbeitsweise vorzuführen und deren Erkenntnispotenzial zu verdeutlichen.

\subsection{Informationen zum Interaktionsausschnitt}

Der zugrundeliegende Ausschnitt stammt aus einer berufsbildenden Schule. Die Videoaufnahmen umfassen zwei Schulstunden à 45 Minuten in einer Berufsfachschulklasse mit Schwerpunkt Metalllehre. Aus zwei Kameraperspektiven (Kamera 1 im Rücken der Schüler, Kamera 2 auf die Klasse gerichtet) wurde eine Doppelstunde im Fach Englisch verfolgt. Es handelt sich um eine rein männliche Berufsfachschulklasse, die aus 17 Schülern im Alter zwischen 15 und 18 Jahren besteht, von denen 13 einen Migrationshintergrund oder eine andere Nationalität haben: Erol (ER) und Özkan (ÖZ) sind Türken, Giovanni (GI) ist Italiener, Marius (MA) hat einen russischen Hintergrund. Der Lehrer, Herr Weber (WE), ist Deutscher. Laut Aussagen des Lehrers ist Deutsch für den Großteil der Klasse bereits die erste Fremdsprache.

Neben der nationalen Heterogenität ist eine relativ geringe Sprachkompetenz in der Fremdsprache Englisch ein weiteres Charakteristikum der Klasse. Dies macht die Erarbeitung von Grundlagen der Grammatik und des Wortschatzes im Unterricht sowie eine häufige Anwendung der Unterrichtssprache Deutsch erforderlich. Das Klassenklima ist offen und der Umgang miteinander respektvoll. Die Schüler haben relativ viel Freiraum für lokale Eigeninitiativen und können das Unterrichtsgeschehen situativ mitgestalten. Passen solche schülerseitigen Initiativen in den Interaktionsverlauf und werden sie in einer ansprechenden und unterhaltsamen Weise realisiert, wird die kurzzeitige Bearbeitung der Schülerrelevanzen vom Lehrer im kontrollierbaren Rahmen zugelassen und ermöglicht (Schmitt 2009).

Die dokumentierte Doppelstunde stand unter dem Thema „Fame and Fortune“. In der zweiten Stunde erstellten die Schüler in einer Stillarbeitsphase jeweils einen Steckbrief einer bekannten Persönlichkeit bzw. die prägnante Beschreibung eines Filmes. Diese Texte wurden vorgelesen und die fragliche Person oder der zur Disposition stehende Film erraten. In dieser Unterrichtsphase ist der Lehrer im Klassenzimmer unterwegs, um sich entweder für Unterstützung anzubieten oder auf Hilfeersuchen einzelner Schüler zu reagieren. Der Lehrer wandert dabei vom äußeren rechten Rand der ersten Tischreihe nach links, um einem Schüler bei einer Frage zu helfen.

\subsection{Der Transkriptausschnitt ${ }^{2}$}

Der Videoausschnitt dokumentiert die Endphase der Erarbeitung der Steckbriefe. Er zeigt den Lehrer, der inzwischen in der letzten Reihe angekommen ist, zwischen den Schülern Justus und Marius platziert. Er hilft zunächst Justus bei der angemessenen englischen Formulierung seines Steckbriefes, wobei der Schüler selbst die Art der Unterstützung anzeigt, die er vom Lehrer wünscht: Ihm fehlen zentrale Vokabeln. Marius nutzt die Anwesenheit des Lehrers bei Justus und die etablierte Unterstützungsaktivität, um den Lehrer zu bitten, seinen ausgearbeiteten Text auf Fehler zu überprüfen (herr weber (-) könnten sie das hier mal durchsehen ob da noch fehler drin). Der Lehrer liest daraufhin den Text und

\footnotetext{
2 Der Ausschnitt ist in Teilen analysiert in Kindermann/Kühner/Schmitt (2011).
} 
beginnt danach mit Marius ein Gespräch darüber. Das Transkript beginnt mit einer viersekündigen Gesprächspause, in der der Lehrer den Schülertext rezipiert und zeigt dann die verbale Interaktion zwischen dem Lehrer (WE) und Marius (MA).

Die folgende Segmentierung folgt der Strukturierung der Beteiligten und charakterisiert sie hinsichtlich dominanter pragmatischer und interaktionsstruktureller Aspekte.

\subsubsection{Implizite Korrektur in der Stillarbeitsphase ${ }^{3}$}

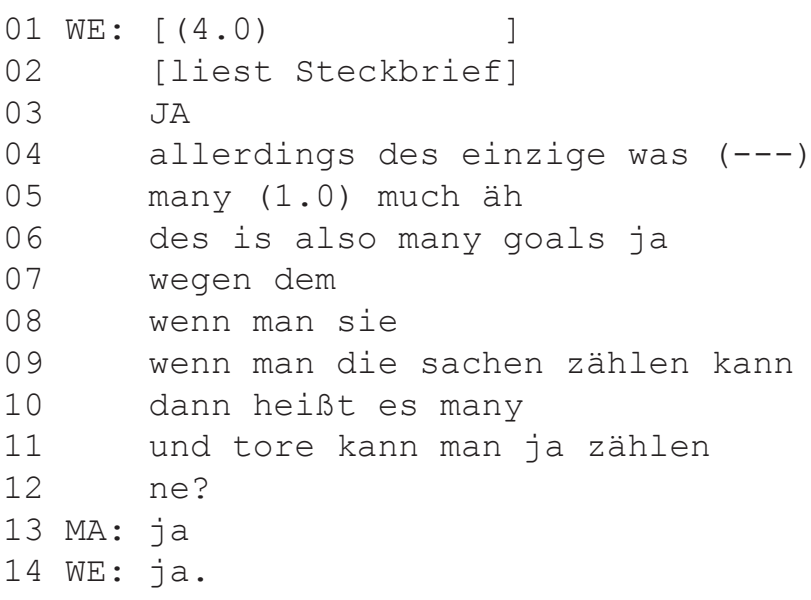

\subsubsection{Unterstützung bei der Orthografie}

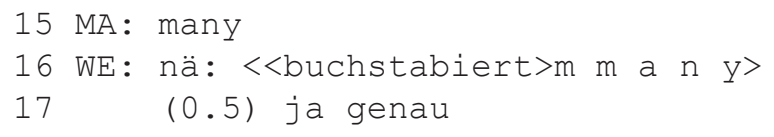

Der Lehrer kontrolliert die Schreibweise und unterstützt Marius bei der korrekten Orthografie.

\subsubsection{Positive Evaluation und Vororientierung des Schülers}

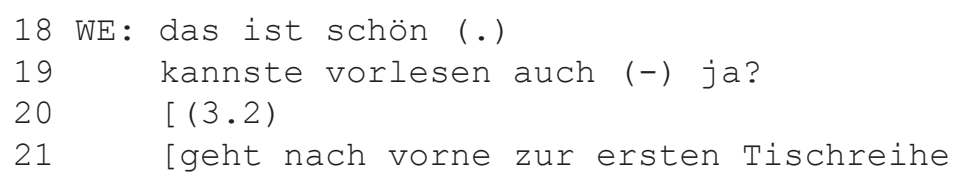

Der Lehrer beendet die Unterstützung von Marius mit einer positiven Evaluation und einer impliziten Ankündigung, den Steckbrief vorzulesen. Er geht danach nach vorne in sein angestammtes Territorium zwischen Tafel und Lehrerpult. Von dort aus eröffnet er das Vorlesen und die Bearbeitung der Steckbriefe.

\subsubsection{Ankündigung der neuen Unterrichtsaktivität}

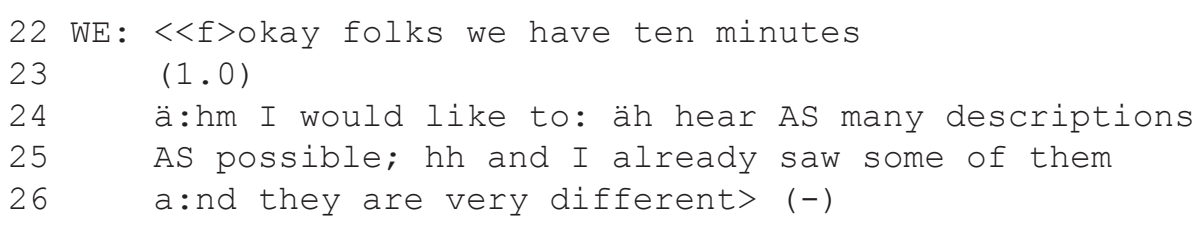

\footnotetext{
3 Die Transkription erfolgt in Anlehnung an GAT 2; siehe Selting et al. (2009).
} 


\subsubsection{Aufruf des ersten Schülers zur Präsentation}

Der Lehrer ruft dann als ersten Schüler Marius auf, dem er kurz zuvor bei der Formulierung seines Steckbriefes geholfen hatte.

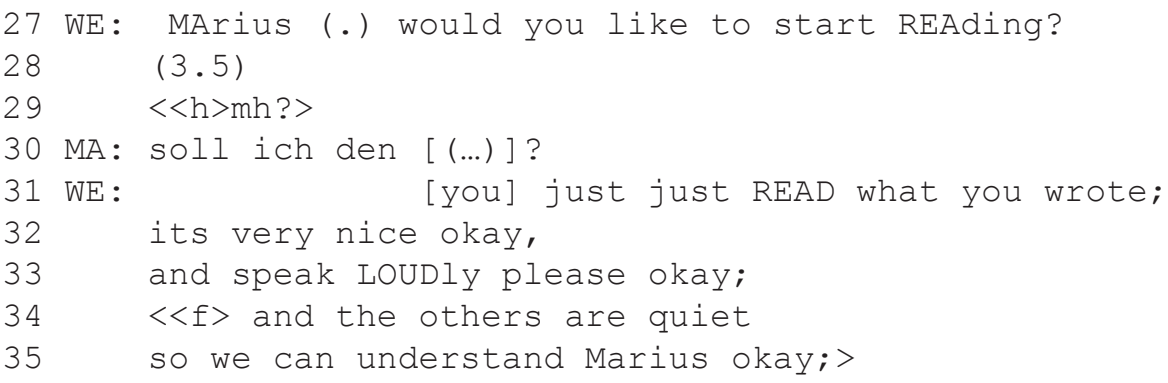

Der Lehrer adressiert den Schüler mit einer Frage und schickt - noch bevor Marius seinen Steckbrief vorgelesen hat - eine positive Evaluation voraus, die auf seiner Kenntnis des Steckbriefes beruht. Dann fordert er den Schüler auf, seinen Steckbrief laut vorzulesen und bittet die restlichen Schüler um Ruhe, damit Marius zu verstehen ist.

\subsubsection{Präsentation des ersten Steckbriefes}

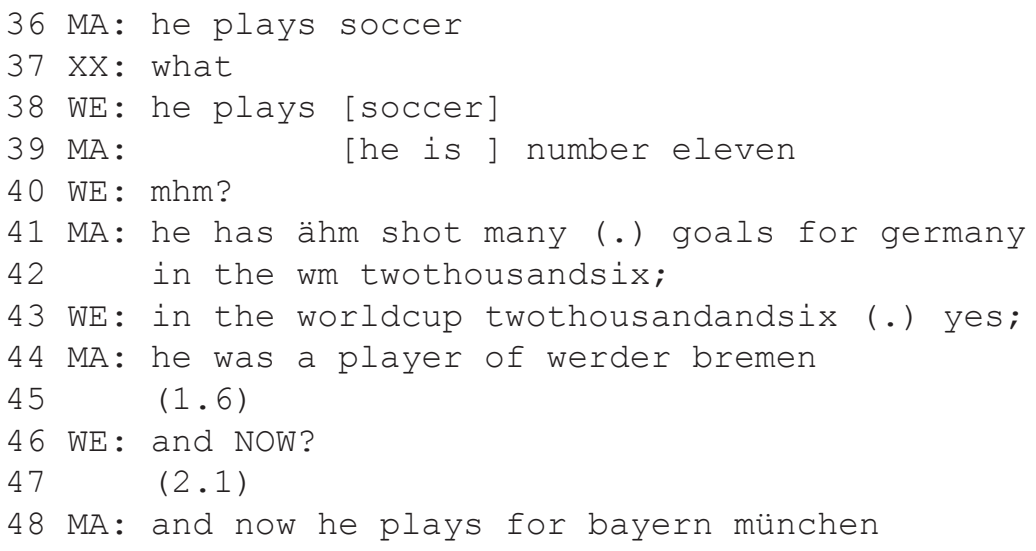

Marius beginnt damit, seinen Steckbrief vorzulesen. Hier ist zu sehen, dass der Lehrer Marius assistiert und die - trotz der Bitte um lautes Sprechen - leise vorgelesenen Informationen in lauter Stimme für die Klassenöffentlichkeit wiederholt. Als Marius die letzte Information seines Steckbriefes vergisst, animiert ihn der Lehrer durch eine Nachfrage, auch diesen Punkt noch vorzulesen.

\subsubsection{Identifikation der Person}

Der Lehrer ruft dann Erol (ER) zur Beantwortung der Frage auf, welche Person sich hinter dem Steckbrief verberge. Erol hat sich bereits frühzeitig zur Beantwortung gemeldet. Erol errät den fraglichen Spieler, bei dem es sich um Miroslav Klose handelt.

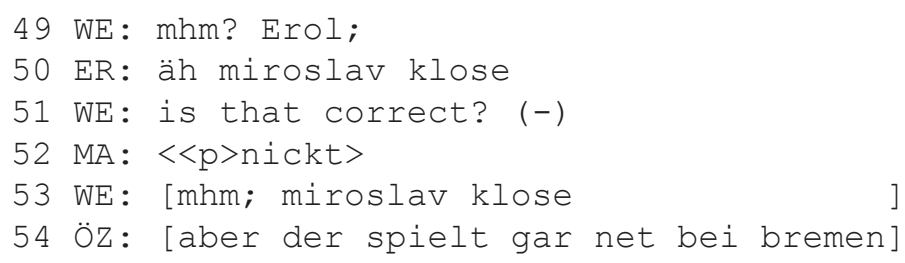




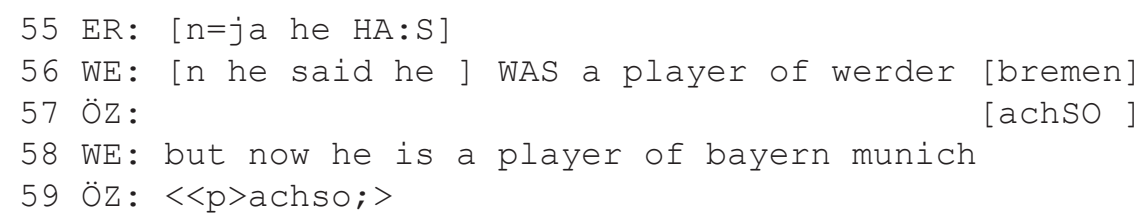

Auf den Hinweis, Klose spiele gar nicht bei Bremen, wiederholt der Lehrer auf Englisch noch einmal die wesentlichen Informationen des präsentierten Steckbriefes.

\subsubsection{Nachlieferung einer Information zur gesuchten Person}

Bevor er den nächsten Schüler aufrufen kann, um den zweiten Steckbrief vorlesen zu lassen, schiebt Erol, der den Spieler erraten hatte, noch eine Information zur Person nach. Der Lehrer korrigiert die Formulierung als inhaltlich richtig, jedoch sprachlich falsch.

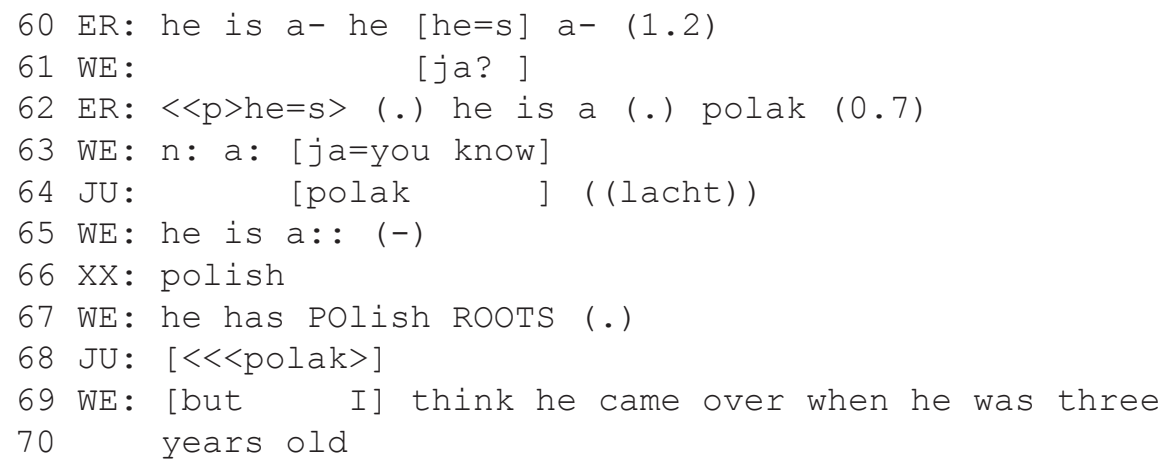

\subsubsection{Etablierung eines alternativen Themas: Ausländer in der Nationalmannschaft}

Dieser Nachschub Erols leitet eine knapp zweiminütige Phase ein, in der sich das Unterrichtsthema „Steckbriefe“ zu einem Diskurs über „Ausländer in der deutschen Nationalmannschaft" entwickelt. Dieser Diskurs wird primär von Giovanni (GI), Özkan (ÖZ) und dem Leher (WE) geführt. Vereinzelt gibt es auch kurze Einwürfe von anderer Seite, die jedoch nicht zugeordnet werden können.

Giovanni etabliert das neue Thema mit dem Hinweis auf die hohe Anzahl von Ausländern, die für die deutsche Nationalmannschaft spielen.

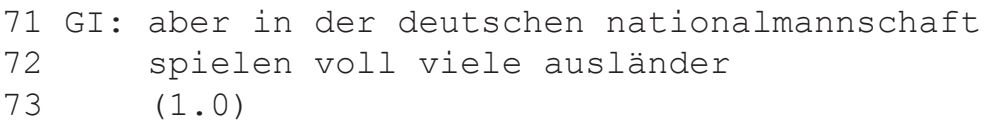

\subsubsection{Problematisierung der Kategorie „Ausländer“ durch den Lehrer}

Der Lehrer reagiert auf diesen Hinweis mit der Problematisierung der Kategorie „Ausländer" und weist darauf hin, dass diese Spieler in Deutschland geboren und somit keine Ausländer, sondern „sozusagen Deutsche“ sind. Özkan wirft ein, ebenfalls hier geboren zu sein, worauf Giovanni mit dem Hinweis reagiert, keinen deutschen Pass zu haben.

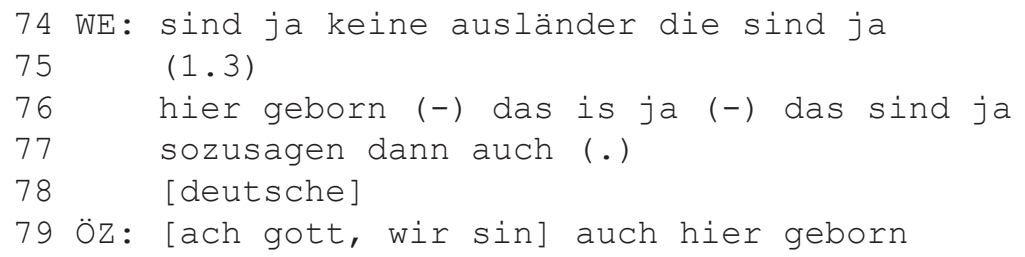


80 WE: ja (-) von daher

81 GI: ja wir ham kein deutsche pass

\subsubsection{Problematisierung der Schülersicht (1)}

Der Lehrer fragt zunächst danach, ob es schlimm sei, dass in der Fußballnationalmannschaft Ausländer spielen. Er übernimmt damit die Kategorie des Schülers. Sowohl Özkan als auch Giovanni beantworten diese Frage zunächst mit einer Zustimmung: Beide finden diesen Sachverhalt problematisch.

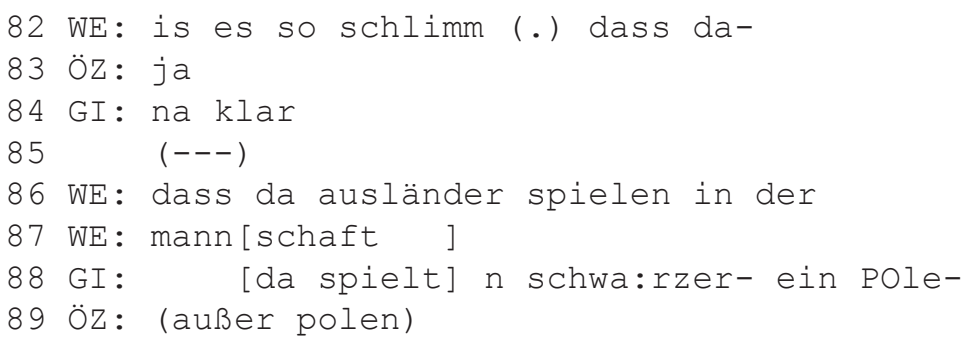

\subsubsection{Problematisierung der Schülersicht (2)}

Der Lehrer unternimmt dann einen zweiten Versuch, die Sicht und Bewertung des Schülers hinsichtlich der in der „Nationalmannschaft spielenden Ausländer“ zu problematisieren. Giovanni verneint dieses Mal, und Özkan verweist darauf, dass auch in Italien ein dunkelhäutiger Spieler für die Nationalmannschaft aktiv ist. Daraufhin entwickelt sich ein Austausch zwischen dem „türkischen“ und dem ,italienischen“ Schüler. Giovanni betont dabei den Ausnahmestatus dieses einen Spielers.

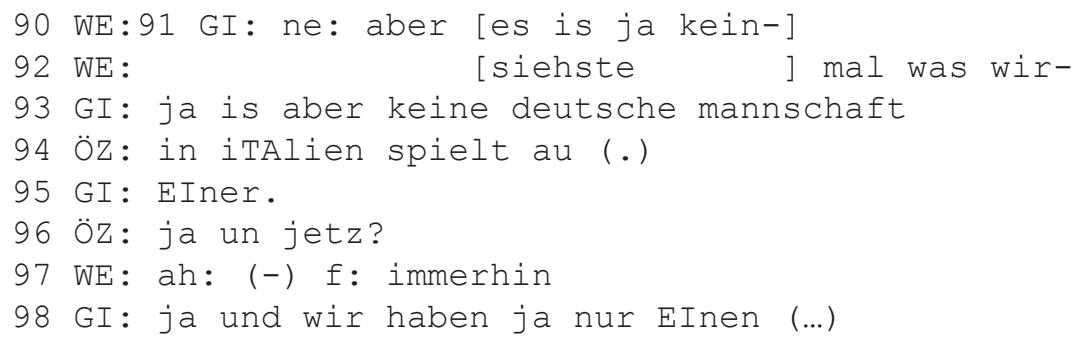

\subsubsection{Ordnungsruf und Rückführung zum Unterrichtsthema}

Der Lehrer unterbricht mit einem Ordnungsruf die Bearbeitung des Themas ,Ausländer in der Nationalmannschaft" und führt mit einer Zusammenfassung des vorgelesenen Steckbriefes wieder zum Thema des Unterrichts zurück.

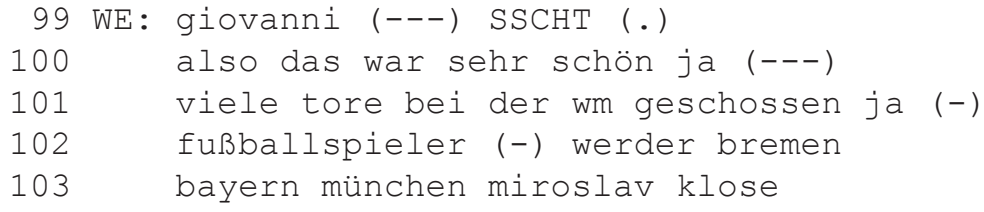




\subsubsection{Nochmaliger Nachschub und Aufruf des zweiten Steckbriefes}

Erol, der zuvor den Spieler erraten hatte, liefert nochmals eine Information zur Person nach, die sich teilweise mit dem Aufruf des nächsten Steckbriefes durch den Lehrer überlappt. Der Lehrer bestätigt die Richtigkeit dieser Information zunächst auf Deutsch, dann in der Unterrichtssprache Englisch.

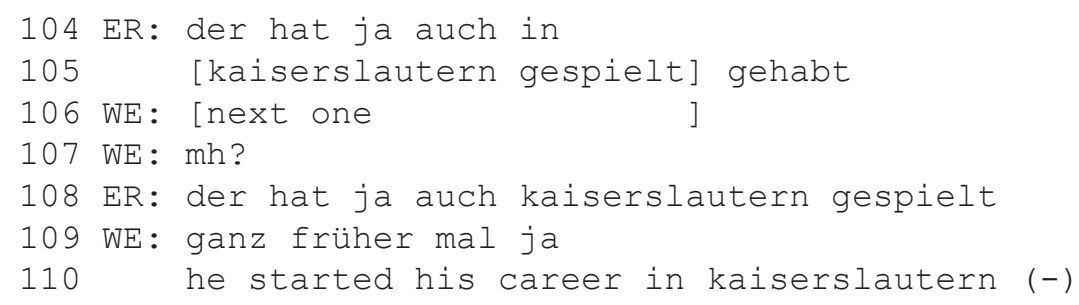

\subsubsection{Aufruf des zweiten Steckbriefes}

Der Lehrer kündigt dann den nächsten Steckbrief an und ruft einen Schüler zum Vorlesen auf.

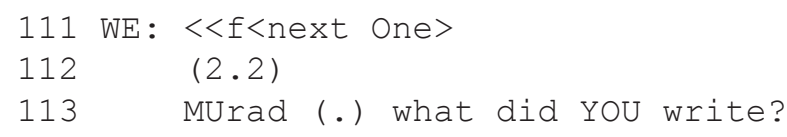

Mit dem dann erfolgreichen Aufruf zum Vorlesen des zweiten Steckbriefes endet der gemeinsame Interaktionsausschnitt. Das Verbal-Transkript und die Strukturbeschreibung des relevanten Kontextes sowie des interaktiven Geschehens stellt die gemeinsame, intersubjektiv gültige und verbindliche Grundlage der nachfolgenden Fallanalysen dar. Falls nötig, wird das Basis-Transkript für die spezifischen Erkenntniszwecke der jeweiligen Perspektive modifiziert und erweitert.

Die Reihenfolge der anschließenden Analysen ist nicht zufällig, sondern orientiert sich an den beiden Aspekten „Komplexität des untersuchten Phänomens“ und „Relevanz ethnografischen Wissens ${ }^{\text {" }}$ für die Durchführung der Analyse: Wir beginnen mit der Perspektive der „Grammatik der gesprochenen Sprache“ (Reinhard Fiehler), gehen dann zur Perspektive der ,interpretativen Soziolinguistik“ über (Ralf Knöbl), der wir die Perspektive der „ethnografischen Interaktionsanalyse“ folgen lassen (Ulrich Reitemeier) und enden mit der Perspektive der „multimodalen Interaktionsanalyse“ (Reinhold Schmitt). Dass es dabei zu einem gewissen Ungleichgewicht bei der Verteilung des zur Verfügung stehenden Raumes kommt, liegt vor allem an der Arbeitsweise der multimodalen Interaktionsanalyse und deren Ergebnispräsentation.

\footnotetext{
4 Die Frage, ob - und vor allem: wann - ethnografisches Wissen in die Rekonstruktionsarbeit eingeführt und nutzbar gemacht werden darf, ist ein Aspekt, der kontrovers diskutiert wird. Die Beantwortung dieser Frage ist ein wesentlicher Aspekt, der die eingangs aufgeführten Ansätze unterscheidet. Zur Bedeutung ethnografischer Informationen für konversationsanalytische Untersuchungen siehe beispielsweise Schegloff (1991, 1992, 1997), Watson/Seiler (1992), Maynard (1989), Cicourel (1992), Billig/Schegloff (1999) und Deppermann (2000).
} 


\section{Literatur}

Billig, Michael/Schegloff, Emanuel A. (1999): Critical Discourse Analysis and Conversation Analysis: An exchange between Michael Billig and Emanuel A. Schegloff. In: Discourse and Society, 10(4), S. 543-582.

Cicourel, Aaron V. (1992): The interpenetration of communicative contexts: Examples from medical encounters. In: Duranti, Alessandro/Goodwin, Charles (Hg.): Rethinking context. Language as an interactive phenomenon. Cambridge: Cambridge University Press. S. 291-310.

Deppermann, Arnulf (2000): Ethnographische Gesprächsanalyse: Zu Nutzen und Notwendigkeit von Ethnographie für die Konversationsanalyse. In: Gesprächsforschung - Online 1, S. 96-124. (Inernet: www.gespraechsforschung-ozs.de):

Fiehler, Reinhard (1985): Einwürfe. In: Sucharowski, Wolfgang (Hg.), S. 77-106.

Garfinkel, Harold (1967): Studies in Ethnomethodology. New Jersey: Prentice Hall, Englewood Cliffs.

Hoffmann, Ludger (1985): Die Bonner Runde - Ansätze zur Analyse einer Kommunikationsform. In: Sucharowski, Wolfgang (Hg.), S. 107-145.

Kindermann, Thorsten/Kühner, Patrizia/Schmitt, Reinhold (2011): Die Bearbeitung eines brisanten Themas im Englischunterricht. In: Schmitt, Reinhold (Hg.): Unterricht ist Interaktion! Analysen zur De-factoDidaktik. Mannheim: IDS. S. 31-67. (= amades 41).

Maynard, Douglas W. (1989): On the ethnography and analysis of discourse in institutional settings. In: Perspectives on Social Problems 1, S. 127-146.

Sacks, Harvey/Schegloff, Emanuel A./Jefferson, Gail (1974): A simplest systematics for the organization of turn-taking for conversation. In: Language 50, S. 696-735.

Sager, Sven (1985): Ein gesprächsanalytisches Schichtmodell dargestellt am Beispiel der Bonner Runde. In: Sucharowski, Wolfgang (Hg.), S. 228-264.

Schegloff, Emanuel A. (1991): Reflections on talk and social structure. In: Boden, Deidre/ Zimmerman, Don (Hg.): Talk and Social Structure. Cambridge: Polity Press. S. 44-70.

Schegloff, Emanuel A. (1992): In another context. In: Duranti, Alesandro/Goodwin, Charles (Hg.): Rethinking context. Language as an interactive phenomenon. Cambridge: Cambridge University Press. S. 191-228.

Schegloff, Emanuel A. (1997): Whose text? Whose context? In: Discourse and Society, S. 165-187.

Schiffrin, Deborah (1994): Approaches to discourse. Oxford, UK/Cambridge, MA: Blackwell.

Schmitt, Reinhold (2009): Schülerseitiges Interaktionsmanagement: Initiativen zwischen supportiver Strukturreproduktion und Subversion. In: Gesprächsforschung - Onlinezeitschrift zur verbalen Interaktion 10, S. 20-69. (Inernet: www.gespraechsforschung-ozs.de/heft2009/ga-schmitt.pdf).

Selting, Margret et al. (2009): Gesprächsanalytisches Transkriptionssystem 2 (GAT 2). In: Gesprächsforschung - Online-Zeitschrift zur verbalen Interaktion 10, S. 353-402. (Inernet: www.gespraechs forschung-ozs.de)

Sucharowski, Wolfgang (Hg.) (1985): Gesprächsforschung im Vergleich. Analysen zur Bonner Runde nach der Hessenwahl 1982. Tübingen: Niemeyer.

Watson, Graham/Seiler, Tom (Hg.) (1992): Text in context. Contributions to ethnomethodology. Newbury Park, CA: Sage.

Dr. Reinhold Schmitt

Institut für Deutsche Sprache

Postfach 101621

D-68016 Mannheim

E-Mail: reinhold.schmitt@ids-mannheim.de 